directly in whole extracts or after fractionation of nucleus and cytoplasm. For the Jcasp model, SET expression was performed by real time PCR. Results: When the Jcasp peptide was internalized, SET moved to the cytoplasm, but no over expression of the protein preceded the delocalization. The delocalized SET was mainly entire and few fragments of small sizes were observed. When the whole SET protein was internalized it localized in the cytoplasm and moved to the nucleus after $3 \mathrm{~h}$. In that case, the protein was mainly entire in the nucleus after $5 \mathrm{~h}$. In each model, when the protein was in the cytoplasm it induced an increase of Tau phosphorylation at epitopes Ser 202, Thr 205, Ser422. Conclusions: The delocalization of SET from the nucleus to the cytoplasm does not require its over expression and/or a previous cleavage of the protein in the nucleus. No additional cleavage of SET is detected when SET is directly internalized in the cytoplasm. The presence of the protein in the cytoplasm is associated to an increase of Tau phosphorylation at sites detected in Alzheimer patient brains, suggesting that the delocalization of SET accelerates the development of the pathology through Tau hyperphosphorylation.

\section{P3-123 WHICH ATTRIBUTES OF TAU MEDIATE TOXICITY: PHOSPHORYLATION OR AGGREGATION?}

Catherine Cowan ${ }^{1}$, Megan Sealey ${ }^{1}$, Douglas Allan ${ }^{2}$, Amritpal Mudher ${ }^{1}$, ${ }^{1}$ University of Southampton, Southampton, United Kingdom; ${ }^{2}$ University of British Columbia, Vancouver, British Columbia, Canada.

Background: We have found that Drosophila which express wild-type human 3-repeat tau (h-3RTau) provide an interesting model of tauopathies such as Alzheimer's disease, and have provided insights into the mechanisms by which tau can cause neuronal dysfunction (Mudher et al., 2004; Chee et al., 2005; Cowan et al., 2010). Flies expressing h-3RTau in their motor neurons have a readily-measurable loco motor behavioral phenotype. This reflects neuronal dysfunction which results from pre-synaptic dysfunction (at the NMJ synapse of motor neuron onto muscle, in this case); which in turn results from defective fast axonal transport in the affected neurons. The defective axonal transport in h-3RTau-expressing animals results from the breakdown of microtubules, which we have measured by transmission electron microscopy. H-3RTau is highly phosphorylated at GSK-3b sites in the fly, and is only weakly able to bind microtubules, as measured by an ex vivo biochemical assay. Remarkably, the presence of h-3RTau also significantly diminishes the ability of the endogenous Drosophila tau (d-Tau) to bind and stabilize microtubules, despite the fact that it fails to form any insoluble tau (such as filaments or tangles) that we have been able to detect. It does this not by sequestering free cytoplasmic unphosphorylated tau into filaments, but by binding it in asoluble complex. This indicates that formation of insoluble tau is not necessary for tau toxicity. Methods: Electron microscopy, locomotion assays, Western blotting. Results: By treating the h-3RT-expressing flies with $\mathrm{LiCl}$ (which has well-known GSK-3b inhibitory activity, as well as other actions) we are able to reduceh-3RTau phosphorylation levels; reduce binding of h-3RTau to d-Tau; improve binding of both h-3RTau and d-Tau to microtubules; restore microtubule integrity; restore axonal transport; and improve locomotion (Mudher et al.,2004; Cowan et al., 2010). Here we show that $\mathrm{LiCl}$ also has the remarkable unexpected effects of increasing total levels of h-3RTau protein, producing insoluble tau, and producing small granular electron-dense structures which might represent granular tau oligomers. We are currently undertaking experiments to determine which of the 3 effects of $\mathrm{LiCl}$ - the reduced tauphosphorylation, the increased tau levels, or the tau aggregate formation - are necessary or sufficient for the observed rescue of tau phenotype. A central question in this set of studies is whether these effects are lithium-specific, and whether they are due to actions of lithium in addition to its GSK-3B inhibition. This is important from amechanistic standpoint, and also because lithium is not a well-tolerated long-term treatment in humans. We explored this using the more specific GSK-3b inhibitor AR-A01448. When we rear Drosophila on media containing $20 \mu \mathrm{M}$ AR-A01448, we reduce phosphorylation of h-3RTau andimprove locomotion just as with $\mathrm{LiCl}$ treatment (Mudher et al., 2004). We now show here that AR-A01448 treatment, like lithium treatment, achieves this rescue by restoring microtubule integrity. Additionally AR-A01448, like lithium, increases levels of h-3RTau protein, and produces $\sim 20$ nmelectron-dense intra-axonal structures which might be insoluble tau. Conclusions: These results indicate that both the expected effects of lithium (in terms of rescuing the tau phenotype) and the unexpected effects of lithium (i.e. increasing the levels of tau protein and producing insoluble tau), are due to GSK-3B inhibition. Our working interpretation of the remarkable fact that two treatments which rescue the tau phenotype also act to increase levels of tau and produce insoluble tau, is that the phosphorylation of tau at key GSK-3b sites is more important in mediating tau toxicity than are tau levels or solubility.

\section{P3-124 EXPLOITING THE DIVERSITY OF THE CHAPERONE REPERTOIRE TO TREAT TAUOPATHIES}

Umesh Jinwal $^{1}$, Jose Abisambra ${ }^{1}$, John Koren ${ }^{1}$, John O’Leary ${ }^{1}$, Laura Blair ${ }^{1}$, Shannon Hill ${ }^{1}$, Justin Trotter ${ }^{1}$, Martin Mushcol ${ }^{1}$, Edwin Weeber ${ }^{1}$, Jason Gestwicki ${ }^{2}$, Chad Dickey ${ }^{1},{ }^{1}$ University of South Florida, Tampa, Florida, United States; ${ }^{2}$ University of Michigan, Ann Arbor, Michigan, United States.

Background: Excessive accumulation of the microtubule associated protein tau in neurons is at the heart of tauopathies, a group of more than 15 diseases that includes Alzheimer's and Parkinson's diseases. Processes that lead to preservation or degradation of tau in the brain are critically linked to the chaperone network. The diverse family of chaperones is likely the most relevant for drug discovery efforts focused on the chaperone system because their inhibition would affect a fewer number of other normal clients, thus reducing the potential for side-effects. We hypothesized that specific chaperone proteins serve a protective role in tau biology by selectively recognizing and delivering aberrant tau for degradation. Methods: Using cell culture techniques, AAV, transgenic mice, and biopohysical approaches we endeavored to explore the effects of chaperone proteins on tau function and pathology. SiRNA, over-expression and mutagenesis have been combined to evaluate the effects of chaperones on tau physiology. Results: We have shown that Hsp90 and Hsp70 each have ATPase activity that can be targeted to regulate the stability of tau. However Hsp90 and Hsp70 are the two primary organizing scaffolds of the chaperone network and may have pleiotropic consequences for other substrates in the cell. Therefore, our group and others have been searching for new targets in the chaperone pathway that might lead to more specific therapeutics. There are more than 150 chaperone proteins critically tied to protein quality control, and these may represent a group of potential therapeutic targets that we are now exploring. Indeed, chaperone proteins like immunophilins and Hsp40s alter the pathophysiology of tau, impacting its clearance as well as its phosphorylation and proteolytic cleavage. Conclusions: We have developed a fundamentally new approach to target discovery for tauopathies by exploring the impact of the molecular chaperone family on tau stability. We predict that identifying these targets will facilitate the development of therapeutics for these neurodegenerative diseases through an interesting and unexplored mechanism: specifically routing the protein quality-control network to handle the tauprotein in a clinically beneficial way.

\section{P3-125 CHARACTERIZATION OF TAU FILAMENT ASSEMBLY WITH THE ADDITION OF VARYING CONCENTRATIONS OF ATP AND GTP USING TRANSMISSION ELECTRON MICROSCOPY}

Mina Farid, Alejandra del Carmen Alonso, Christopher Corbo, Maria Eugenia Alaniz, CUNY College of Staten Island, Staten island, New York, United States.

Background: It is pivotal to understand the mechanistic function of synaptic breakdown that precedes in cell death in Alzheimer's disease and other neurodegenerative diseases. One of the causes of neurodegenerative diseases is a dysfunctional tau protein. Accumulation of hyperphosphorylated tau causes the disruption of microtubules; which are related to synaptic loss and pathology of Alzheimer's disease. Impaired cognitive function and pathology of $\mathrm{AD}$ is correlated with this lesion. We have previously shown that the cytosolic Alzheimer hyperphosphorylated tau (ADP-tau) sequesters normal tau, MAP1A, MAP1B and MAP2, which results in the inhibition of 